\title{
The RASSCF, RASSI, and CASPT2 Methods Used on Small Molecules of Astrophysical Interest
}

\author{
Per-Ake Malmqvist \\ Theoretical Chemistry, Chemical Center, \\ P.O. Box 124, 22100 Lund, Sweden
}

\section{Introduction}

To a quantum chemist with no particular background in astrophysics or astronomy, a brief glance at journals and textbooks in these fields shows at least three areas where computational quantum chemistry has had a valuable impact: Interstellar cloud chemistry; stellar atmosphere modelling; and chemistry in extreme conditions, such as at the surface of a neutron star. The first two uses are particularly suitable, since standard methods are directly applicable.

For such problems, good calculations of potential energy as well as expectation values and matrix elements of dipole and other operators appears to be in demand. Many electronic states may be involved, at least a broad range of problems involve fairly small molecules, often radicals, and conformation regions far from equilibrium. Such problems are addressed by three methods originated in our laboratory, and known by the acronyms RASSCF (Restricted Active Space Self-Consistent Field, Malmqvist et al. 1990), RASSI (RAS State Interaction) and CASPT2 (Complete Active Space Perturbation Theory to Second OrderComplete Active Space Perturbation Theory to Second Order, Andersson et al. 1990; Andersson et al. 1992).

The first method is an extension of the well-known CASSCF (Complete Active Space SCF) method (Roos et al. 1980; Roos 1980). The RASSI is a versatile analysis method, (an extension of CASSI; Malmqvist \& Roos 1989), whereby separately optimized RASSCF wave functions can be used as basis functions, for computing transition moments, defining diabatic states, etc. Finally, the CASPT2 method uses a CASSCF wave function as a multiconfigurational zeroth order wave function, and corrects for dynamic correlation to second order in energy. In contrast to most conventional perturbation techniques, it works well for arbitrary spin and symmetry, for excited states, etc., without any particular demands on the electronic structure of the root function.

A rough sketch of computational quantum chemistry methods will be given, and against this background the methods will be briefly described with demonstration applications. 


\subsection{The Basic Problem: The Adiabatic Electronic Structure}

Quantum chemical problems are concerned with some aspect of the solution of the Schrödinger equation applied to a system of nuclei and electrons. The electrodynamic field is imposed only as an external field. Approximate relativistic effects, and nuclear size effects, are incorporated by modifying the interactions. Any more sophisticated effects are added as corrections computed by perturbation theory. The time-dependent Schrödinger equation, with nuclear and electronic coordinates $\mathbf{Q}$ and $\mathbf{q}$, resp., reads

$$
\left.\begin{array}{rl}
i \frac{\partial}{\partial t} \Psi(\mathbf{q}, \mathbf{Q}, t) & =\hat{H} \Psi(\mathbf{q}, \mathbf{Q}, t) \\
\hat{H} & =\hat{T}_{n}+\hat{T}_{e}+\hat{V}_{n n}+\hat{V}_{e e}+\hat{V}_{e n}
\end{array}\right\}
$$

where the second line simply sums the contributions from kinetic energy of nuclei and electrons, the interaction among nuclei, among the electrons, and between nuclei and electrons, respectively. Those terms may include relativistic corrections, and are believed to account for the physics of this finite system to a precision well in excess of that needed for all chemical and almost all spectroscopic applications.

Practical solution of this equation preceeds in two steps. The hamiltonian is split into $\hat{H}=\hat{T}_{n}+\hat{H}_{e}$. All terms (including nuclear-nuclear repulsion) that commute with the nuclear position operators have been included in the electronic hamiltonian $\hat{H}_{e}(\mathbf{Q})$, which is thus an operator on electronic wave functions as function of the nuclear positions. Expanding $\hat{H}_{e}(\mathbf{Q})$ in a complete set of electronic basis functions, which depend on the nuclear coordinates, the full Schrödinger equation takes a form that involves only nuclei.

A complete basis is never available. However, a practical and almost always sufficient basis is provided by the few lowest eigenfunctions of $\hat{H}_{e}(\mathbf{Q})$; this is called the adiabatic basis, and the Schrödinger equation becomes

$$
\begin{aligned}
i \frac{\partial}{\partial t} \chi_{K}= & -\frac{1}{2 M} \frac{\partial^{2}}{\partial Q^{2}} \chi_{K}+V_{K}(Q) \chi_{K}-\frac{1}{M} \sum_{L}\left\langle\Phi_{K} \mid \frac{\partial}{\partial Q} \Phi_{L}\right\rangle \frac{\partial}{\partial Q} \chi_{L} \\
& -\frac{1}{2 M} \sum_{L}\left\langle\Phi_{K} \mid \frac{\partial^{2}}{\partial Q^{2}} \Phi_{L}\right\rangle \chi_{L}
\end{aligned}
$$

where $\Phi_{K}(\mathbf{q}, \mathbf{Q})$ and $\chi_{K}(\mathbf{Q}, t)$ are the electronic and nuclear wave functions. The potential energy functions for the nuclear motion are just the energy eigenvalues of $\hat{H}_{e}(\mathbf{Q})$.

So far, the treatment is formally exact. If the two matrix elements involving derivatives with respect to nuclear coordinates are neglected, we obtain the BornOppenheimer (BO) approximation. When this is not valid, the equation can in practice still be solved using a few selected adiabatic wave functions as basis.

Disregarding exceptional cases where non-BO effects must be included already from the beginning, we see that the problem has a very nice modularity: We first solve the electronic problem for relevant eigenstates. The result provides input for a number of other methods, which perform e.g. reactive scattering or 
ro-vibrational analysis. Such calculations have their own broad range of methodology and is also a part of the quantum chemistry repertoir. However, we will keep within the scope of electronic eigenstates.

The basic requirement of quantum chemistry methods is then the ability to obtain, for any conformation of nuclei, the adiabatic electronic energy, and electronic matrix elements of various fairly simple operators, of any of the lower electronic eigenstates. The required differential accuracy depends on the problem, but is typically a few $\mathrm{kJ} / \mathrm{kmol}$ in potential energy functions. Much higher accuracy can be demanded in spectroscopic applications, but may still be reached when errors cancel, or when matrix elements for small perturbations are wanted. Spectroscopy usually requires matrix elements of a broader range of operators. From a quantum chemist's viewpoint, the basic problems are similar.

The general applicability, with a well-balanced description of different electronic structures, is a tall order. One cannot expect any single method to solve all problems. As a consequence, a large number of methods are used to obtain electronic energies. In the following, a brief overview is offered, on the understanding that it is not complete, and in some cases not well-informed as regards latest research status.

\section{Quantum Chemistry Methods in General}

Those methods which compute electronic eigenstate energies and properties can be subdivided into density functional and wave function methods, and the latter into configuration based methods (single- vs. multi-configurational) and explicitly correlated (" $r_{12}$ "-) methods. This subdivision is ordered by an increasingly detailed description of the system, and thus an increasing accuracy but also a rapidly increasing computation cost.

Density functional theory expresses the minimum electronic energy for any specified electronic charge density function in the form of a so-called energy functional. The exact functional is not known in any practically useful formulation, and its existence is just used as a justification for a variety of pragmatic approximations. Recent improvements have made such methods useful for molecules, and since they are cheap and scale very favourably with molecular size, they may well be the only practical alternative for many problems. A number of useful references can be found, e.g., in Clementi et al. 1991. A drawback is the lack of any systematic way to improve the result by larger calculations, and of a good indicator of the reliability of any specific result.

Response and transition properties can also be computed by a range of other methods, which will only be mentioned in passing: In general, they use wave functions calculated by a specific wave function method, and use some of the theoretical and computational machinery of that method to obtain transition strengths and energies, ionization potentials etc. The accuracy in transition properties can surpass that obtained from simple matrix elements over the separately computed state functions. 
The end result of methods described below is in general a set of potential energies and various electronic matrix elements, which are then expressed as parametrized functions of internal coordinates.

\subsection{Wave Function Methods}

Excepting density functional methods, we compute an approximation to the wave function. This is defined in terms of a large number of parameters, whose meaning are defined by some wave function model. The parameter values are optimized by requiring key properties of the exact wave function to be exactly represented in the model: usually the stationarity criterion for an energy eigenstate. The model should be able to reproduce the most important features of the exact wave function. A list of such features, in approximate order of diminishing importance, could be:

1. A major part (overlapping by about $0.985^{n}$, if $\mathrm{n}$ is the number of electrons, with the exact wave function) requires a few (typically 1-100) so-called determinant functions, if those are properly chosen. At specific conformations, non-radicals may need only 1 (SCF). Dissociation or reaction paths require more. The entire chemistry, i.e. the set of all reaction and dissociation paths, for even a small number of atoms is rarely done, but may require thousands of determinants. A couple of hundred are needed to dissociate the $\mathrm{Cr}_{2}$ molecule. When more than one configuration is important, one speaks of static correlation, or near-degeneracy effects. Other correlation effects are then called dynamic correlation.

2. When an electron is close to a nucleus, the wave function has an electronnuclear cusp.

3. When it is far away from the rest of the molecule, the wave function goes asymptotically to describe the ground state of the kation, properly symmetry coupled to an orbital with exponential tail describing the remote electron.

4. The major effect of varying the position of one electron is to polarize the remaining electron system. This effect may be called dynamic polarization, or intramolecular dispersion. It affects most strongly the immediate vicinity of the electron, but is fairly long-range. It affects quite strongly also the core electrons, and the sigma skeleton of conjugated molecules, which are traditionally though of as rather inactive.

5. Apart from this polarization, there is also an inter-electron cusp. This is of small importance to the energy, but affects some two-electron properties.

In addition, it is usually quite important that dissociation gives asymptotically a properly spin- and symmetry-coupled sum of products, with factors that are energy eigenstates of the respective fragments (Size consistency).

There are of course methods designed to solve one-, two-, or three-electron problems, which use specialized forms for the wave function. Excepting these, the universal ansatz is an expansion in Slater Determinant Functions (SDs) or Configuration State Functions (CSFs). The SDs are antisymmetrized products 
of one-electron functions; the CSFs are linear combinations of SDs which have desired total spin and spatial symmetry. The expansion coefficients can be explicitly used as variation parameters, or implicitly defined as in Coupled Cluster or perturbation theory.

On top of this expansion, correlation factors which depend explicitly on the interelectron distances can be used to introduce the interelectronic cusp, which improves the convergence rate of CSF or perturbation expansions, and which is crucial to sampling methods such as Quantum Monte Carlo. The latter type of methods are able to generate millions of electron positions statistically drawn from an ensemble that accurately represents the ground state wave function, and form a Monte-Carlo estimate of the energy. However, mainstream methods rely only on CSF expansions, where local features are very poorly represented, but where energies and most properties are obtained by integrals which are not very sensitive to these defects.

\subsection{Configuration Expansion Methods: Orbital Generation}

All methods considered here rely on a one-electron basis set, which must be able to reproduce the cusp and tail of items 2 and 3 above. This would seem to favor STO basis sets, with exponential functions similar to hydrogenic wave functions. However, contracted GTO bases, which are sums of gaussian functions instead, can easily fulfill these requirements in the $\mathcal{L}^{2}$ sense, and are much easier to handle. For accurate pointwise evaluation of wave function (QMC) or density there is some justification for other bases in molecular problems, and then a better approach is to use some modifying factor times a standard gaussian basis set. The selected basis set, the "AO basis", is used to express molecular orbitals, or MO's. Modern basis sets are usually general contractions of the ANO type (Atomic Natural Orbitạls, Almlöf \& Taylor 1987).

Determination of suitable MO coefficients is done by SCF or MCSCF. One or more Slater Determinant functions (SD), or Configuration State Functions (CSF) are formed from the MOs. For any given such function, those MOs which are used to define it are called occupied, the other are unoccupied. The MO coefficients, describing the orbitals, are used as variational parameters. If more than one SD or CSF is used, there are in addition CI (Configuration Interaction) coefficients to express the wave function. The MO and CI coefficients are varied to find a stationary energy expectation value. The usual orbital generation methods are:

1. UHF ("Unrestricted" HF). The spin-orbitals are restricted to have either spin up or down $(\alpha$ or $\beta)$.

2. RHF (Restricted HF): All orbitals come in pairs, with $\alpha$ and $\beta$ spin but the same spatial dependence. They do not have to be occupied in pairs:

3. High-Spin RHF: All singly occupied orbitals have $\alpha$ spin.

4. OSHF (Open-Shell HF): A spin- and symmetry-coupled CSF is being optimized. This is rarely done nowadays, except in the High-Spin special case, where the CSF is a single SD. 
5. MCSCF (Multi-Configurational Self-Consistent Field): A wave function composed of several or many CSFs is optimized.

In a basis set approach, the orbital generator produces not only the set of occupied orbitals, but a complete set within the basis. Those which are doubly occupied in all configurations are inactive orbitals. Those that are never used are virtual, or secondary, orbitals. Those with varying occupancy in MCSCF, or open shells, are active orbitals.

\subsection{Correlation by CSF Expansion}

The SCF and MCSCF techniques give energies and properties which are often useful in their own right. In particular, for few-electron systems the MCSCF can include enough configurations to account not only for static correlation, but for intramolecular dispersion and some close-range dynamic correlation as well. The advantage is then that the orbitals are optimized together with the correlation treatment. To afford large-scale MCSCF for more than a few electrons, one must select the CSFs used as economically as possible. However, this is not easy when the selection is to be valid for several or many conformations and states. Moreover, such general MCSCF is plagued with existence of multiple solutions to the stationarity conditions. The most popular form of MCSCF is therefore the CASSCF (Complete Active Space) method, where all the CSFs possible with a given active space are included. Their number increases as a polynomial with the number of active orbitals, with leading degree equal to the number of active electrons. This dependence sets a sharp limit to the possibility of including dynamic correlation by merely increasing the active space. The CASSCF is thus followed by a calculation of remaining correlation energy by CI-like or nonCI-like methods. With the former, I mean the well-known SDCI (Singles and Doubles CI) with single- or multi-configuration reference (MR-SDCI, or MRCI for short), and to methods which technically are simple modifications to SDCI: $\mathrm{CPF}$ and MCPF ([Modified] Coupled Pair Functional) in the single-configuration case, ACPF (Average CPF) in the multi-configuration case (Ahlrichs et al. 1985; Chong \& Langhoff 1986; Gdanitz \& Ahlrichs 1988).

With these methods, wave functions are obtained in the CSF space generated by substituting at most $t$ wo orbitals in the reference configuration(s). The SDCI is not size-consistent, so the modifications are preferred if many electrons are correlated. With the above methods, properties are easily obtained. Transition matrix elements are easily obtained only by the SDCI method, and assuming a common orbital basis for the states.

With non-CI-like methods, there is a large difference between the singleand multi-configuration treatments. In the single-configuration case, there is a wide range of different methods, with programs available and useful also for non-specialists. This is especially true for RHF-based methods for non-radicals (which could, technically speking, also be considered as Cl-like). High-spin open shell cases can be treated as UHF, with some annoying loss of efficiency, while OSHF references are on their way but not yet firmly established. The most 
important methods are MP2 (Second-order Møller-Plesset), MBPT4 (Fourthorder Many-Body Perturbation Theory), and CC (Coupled Cluster). The latter usually includes single and double excitations in its parameter space (CCSD), but unless the number of orbitals is large, triples can be included approximately or exactly (CCSD(T), CCSDT) to yield very accurate results, whenever a single closed-shell reference is adequate. A good application example with important references is the article by Watts et al. 1991. By contrast, the MP2 is a very popular method for larger molecules, since it is fairly cheap and can be used without storing the electron repulsion integrals.

With a multi-configuration reference space, the only successful general scheme so far is to use a single reference function composed of many configurations, of the CASSCF type, as the root function of a perturbation expansion.

\section{The RASSCF, RASSI, and CASPT2 Methods}

\subsection{R.ASSCF}

The major drawback of CASSCF is the steep increase in the number of CSFs with the number of active orbitals, except when very few electrons are correlated. With twelve active electrons, there is a limit at around twelve or thirteen active orbitals, which give 226512 and 736164 singlet CSFs, respectively. There are many examples where such an active space is not sufficient for obtaining good CASSCF energies and properties, and quite a few examples where not even the CASSCF orbitals are good enough to be used in subsequent MRCI or similar methods. This happens when the dynamic correlation, lacking in the CASSCF, is important for determining the shape of the orbitals. A typical example is negative ions, where lack of dynamic correlation gives too diffuse orbitals, or when dynamic correlation alters the balance between competing electronic structures with large and different dipole moments. General MCSCF is not an attractive scheme, since it is difficult to preselect the important configurations, and to avoid multiple minima.

The name Restricted Active Space was given by Olsen (Olsen et al. 1988) to a scheme where the active orbitals are subdivided into RAS-1, RAS-2, and RAS-3 orbitals. All CSFs are included, which have at most a specified number of electrons in the RAS-3 space, and at most a specified number of electrons taken out from the RAS- 1 space. In RAS- 2 orbitals, any occupation is allowed. The restrictions on RAS-1 and RAS- 3 can cut down the number of CSFs quite drastically, thus allowing a larger active space, and are based entirely on an orbital selection which is easier and more natural than a general CSF selection.

The RAS expansion space is closed under deexcitation, which is a technically important property. It ensures that any matrix elements expressed by spin-free second quantization formulae can, after minor rearrangement, be expressed by means of vector transformations within the expansion space. This makes it effcient to regard the application of a linear operator as a programming primitive. Our RASSCF code first used the Split-Graph GUGA method for operations on 
the CSF expansion (Malmqvist et al. 1990). In a later version such operations are performed by temporarily reexpanding in SDs and, after the operations, transform back to CSFs.

Leaving out any technicalities, the program is simple: Given a set of starting orbitals, the CI equations are solved for the state of interest. For this given CI expansion, the orbitals are refined so that the energy is stationary. If this scheme is repeated as it stands, convergence may suffer from neglect of the coupling between the CI- and the orbital-optimization steps. However, this coupling is introduced in all subsequent iterations by a correction, which is refined at each iteration. A number of variants are possible, such as a weighted optimization of several states simultaneously. The end result is orbitals, wave functions, density matrices, properties and matrix elements for the RASSCF states.

\subsection{RASSI}

Since its CSF space is closed under deexcitation, a RASSCF wave function can also be reexpressed in any new orbital set, provided that the transformation matrix is triangular (or even block-triangular with the subdivision into inactive,RAS-1, etc. orbitals). The CI expansion coefficients are easily and efficiently recomputed to express identically the same wave function in the new orbital set. The new orbitals will no longer be orthonormal. The RASSI program puts this to use by accepting as input two different RASSCF wave functions, each with different orbitals, and proceeds by performing such non-orthogonal orbital transformations that the resulting two new orbital sets $\left\{\psi_{p}^{A}\right\}$ and $\left\{\psi_{q}^{B}\right\}$ are biorthonormal:

$$
\left\langle\psi_{p}^{A} \mid \psi_{q}^{B}\right\rangle=\delta_{p q}, \quad \forall p, q .
$$

The point is that with such orbitals, any matrix element can be computed by standard methods: If an operator is expressed by a second quantization formula with integrals over the mixed new basis, the expression for its matrix element between states $\mathrm{A}$ and $\mathrm{B}$ is identical to the form it would take in an common orthonormal basis.

The RASSI program is extensively used just to compute standard one-electron matrix elements of e.g. the dipole operator, for oscillator strengths (See the description of its precursor, CASSI, in Malmqvist \& Roos 1989). With state-specific orbitals, such matrix elements cannot be obtained from dynamic correlation post-RASSCF programs, and the RASSCF values computed by RASSI is then our only source of oscillator strengths. When the states have the same symmetry, the RASSCF states may well be non-orthogonal and interacting. However, the RASSI program can simply solve the Schrödinger eigenvalue problem in the basis of any provided RASSCF wave functions, thus yielding oscillator strenghts for orthogonal and non-interacting RASSI states instead. The difference may be quite important, when two RASSCF states have different permanent dipole moment: The transition dipole, if evaluated for the RASSCF states, is contaminated by the product of the overlap with the difference in dipole moment.

The RASSI program is also very helpful in a variety of situations: Even if the RASSCF is simpler than general MCSCF, it is sometimes difficult to select 
orbital subspaces properly. Two putative different solutions may actually be misrepresentations of the same physical state, if the optimization has resulted in correlation of either of two bonds for instance, when the subdivision of the active space prevent them from being treated democratically. Occasionally, there are even problems with spontaneous symmetry breaking. This type of problems may be resolved by simply giving all the available RASSCF states as input to RASSI and solve the Schrödinger equation in the space they span. The result will in general be a number of reliable RASSI states, and their natural orbitals will be excellent start orbitals for new state-specific calculations, while their natural occupancies will indicate how to pick the new active orbitals. The original RASSCF wave functions will be reexpressed in the orthonormal, non-interacting RASSI states, which indicates in which way the states have perturbed each other.

\subsection{CASPT2}

When RASSCF does not give sufficient dynamic correlation, the preferred additional procedure is $\mathrm{MRCI}$, if it can be afforded. If more than a few electrons are correlated, it must be corrected for size consistency or replaced by its cousin ACPF. However, it is necessary that the reference selection of CSFs is large enough. It must contain a large fraction of the wave function, and a similar fraction for all states and conformations. It must be realized that when going from CASSCF or RASSCF to MRCI, we are not just adding a large number of important configurations, but also deleting by selection, and perhaps more severe: One part, with a weight of e.g. around $85 \%$, of the CASSCF wave function, is now improved by dynamic correlation; $15 \%$ is not. These two parts may differ considerably in ionizity, dipole moment etc, and are not treated in a balanced way, which will affect the result. In fact, whenever possible, the best procedure is to use several references, and extrapolate the results, as function of a selection threshold. For more than a few atoms, or when large references are necessary, MRCI may be too expensive. The CASPT2 method was developed to account for extra-CAS dynamic correlation, when a satisfactory MRCI treatment is too expensive (Andersson et al. 1990; Andersson et al. 1992). However, it is not an approximation to MRCI, but a (size-consistent) perturbative approximation to Full CI, and uses the complete CASSCF wave function as reference.

The CASPT2 program solves the traditional first-order Rayleigh-Schrödinger perturbation equations with a Møller-Plesset splitting of the Hamiltonian:

$$
\left.\begin{array}{rl}
\hat{H} & =\dot{H}^{(0)}+\hat{H}^{(1)} \\
\left(\hat{H}^{(0)}-E^{(0)}\right) \Psi^{(1)} & =-\hat{H}^{(1)} \Psi^{(0)} \\
\hat{H}^{(0)}-E^{(0)} & =\hat{Q}(\hat{F}-\text { const. }) \hat{Q} \\
\hat{F} & =\sum_{p q} F_{p q} \hat{E}_{p q}
\end{array}\right\}
$$

where $\hat{Q}$ is a projector onto the interacting space of $\Psi^{(0)}$, which is a CASSCF wave function. The Fock-like matrix $F_{p q}$ is defined such that it gives the usual Fock matrix for a closed-shell reference function, and fulfills Koopmans' theorem 
for ionization energy and electron affinity in an average sense for the active orbitals. The equation is solved by expanding in the interacting space:

$$
\Psi^{(1)}=\sum_{p q r s} C_{p q r s} \hat{E}_{p q r s} \Psi^{(0)}
$$

and the correlation energy is obtained to second order as

$$
E^{(2)}=-\left\langle\Psi^{(1)} \mid \hat{H}^{(0)} \Psi^{(1)}\right\rangle
$$

In these equations, $\hat{E}_{p q r s}$ is an excitation operator, which effects a spincoupled excitation of two electrons from orbitals $q$ and $s$ to orbitals $p$ and $r$. For a CASSCF reference, the meaningful excitations are those where $q$ and $s$ are inactive or active, $\mathrm{p}$ and $\mathrm{r}$ are active or secondary, but all four indices are not active. Of the eight different cases, the most expensive terms arise from the semi-internal excitations, where three of the orbital indices pqrs refer to active orbitals. To solve the resulting equations requires then essentially the threebody density matrix elements for active orbitals, which are reasonably easy to compute but gives equation matrices of the size $n^{3} \times n^{3}$. This causes a calculation bottleneck for more than about $n=12$ active orbitals. The calculation then scales as fast as $n^{9}$ in the present algorithm, which is the main reason why the method has not yet been worthwhile to append to RASSCF rather than CASSCF.

It should be noted that the present implementation is completely independent of the number of CSFs, apart from the initial, rapid evaluation of the density matrix elements. It has been used with equal ease for single configuration root functions as with CASSCF wave functions with a hundred thousand configurations. The interacting space can easily comprise billions of CSFs; the number of variational parameters still scale quite modestly, and is slightly larger than with a similar closed-shell MP-2 calculation.

\section{Demonstration Examples}

\subsection{RASSCF}

Ethene V state. The first singlet $\pi \pi^{*}$ state of ethene, the so-called V state, is an example where a meaningful orbital optimization must include dynamic correlation. The state is dominated by two valence-bond structures, each with one positive and one negative methylene ion. It has been much studied as a prototype example of high ionicity states. Unless enough dynamic correlation is included, the orbitals will be too diffuse. Table 1 illustrates the very rapid growth of the number of CSFs in an ill-advised attempt to obtain good orbitals by CASSCF (In the original study, another approach was taken, of course: MRCI with iterated natural orbitals).

By contrast, in the last line is a RASSCF calculation with 29 active orbitals, requiring a very modest number of CSFs. The total energy is much lower, of course, but this is a dangerous quality measure in this case. The fourth column 
Table 1. RASSCF demonstration example: Ethene

\begin{tabular}{lrrrr}
\hline Calculation & \# CSFs & $\begin{array}{c}\text { Energy } \\
(\text { a.u. })\end{array}$ & $\begin{array}{c}\left\langle z^{2}\right\rangle \\
(\text { a.u. })\end{array}$ & $\begin{array}{r}\text { CPU } \\
(\mathrm{s})\end{array}$ \\
\hline CAS $(10 \sigma, 2 \pi)$ & 28848 & -77.8993 & 22.8 & 15 \\
CAS $(10 \sigma, 3 \pi)$ & 92406 & -77.9239 & 23.4 & 60 \\
CAS $(10 \sigma, 4 \pi)$ & 268800 & -77.9341 & 23.0 & 227 \\
CAS $(10 \sigma, 5 \pi)$ & 716523 & -77.9433 & 21.9 & 878 \\
RAS $(5,2,22)$ & 4704 & -78.0484 & 20.8 & 68 \\
\hline
\end{tabular}

is the expectation value of $\sum z_{i}^{2}$. Its true value is believed to be around 15 a.u., and the excess is primarily due to the $\pi^{*}$ orbital which is forced to be too diffuse. All calculations correlate the 12 valence electrons. The basis set is of ANO type, with $6 \mathrm{~s}, 5 \mathrm{p}, 3 \mathrm{~d}$ and $2 \mathrm{f}$ functions on carbons, $3 \mathrm{~s}$ and $2 \mathrm{p}$ functions on hydrogens. The CPU time is for an IBM 3090-17S computer.

The radiative lifetime of $\mathrm{SH}^{+} \boldsymbol{A}^{3} \boldsymbol{\Pi}$. Here, the potential and transition dipole functions of the ground $X^{1} \Sigma^{+}$and the $A^{3} \Pi$ states have been computed by RASSCF (González-Luque et al. 1992). The spectroscopic constants derived for the two states are presented in Tabs. 2 and 3 , compared to experiment (Rostas et al. 1984).

Table 2. The $\mathrm{SH}^{+} X^{3} \Sigma^{-}$state.

\begin{tabular}{lllll}
\hline & \multicolumn{2}{c}{ Normal } & \multicolumn{2}{c}{ Deuterated } \\
Quantity & \multicolumn{1}{c}{ RAS } & Exp & RAS & Exp \\
\hline$R_{e}$ & $1.367 \AA$ & 1.364 & 1.367 & - \\
$D_{e}$ & $3.46 \mathrm{eV}$ & 3.70 & 3.46 & - \\
$D_{0}$ & $3.30 \mathrm{eV}$ & 3.54 & 3.35 & 3.59 \\
$\omega_{e}$ & $2540 \mathrm{~cm}^{-1}$ & 2548 & 1824 & 1830 \\
$\omega_{e} x_{e}$ & $51.7 \mathrm{~cm}^{-1}$ & 49.3 & 26.8 & 25.4 \\
$B_{e}$ & $9.24 \mathrm{~cm}^{-1}$ & 9.28 & 4.76 & 4.79 \\
$\alpha_{e}$ & $0.30 \mathrm{~cm}^{-1}$ & 0.28 & 0.11 & 0.11 \\
\hline
\end{tabular}

The end results for the life time is $1.07 \pm 0.13 \mu \mathrm{s}$ and $1.14 \pm 0.13 \mu \mathrm{s}$, in excellent agreement with one experiment but in disagreement (but within error bars) with another. For details, we refer to the original article which also quotes other similar examples. 
Table 3. The $\mathrm{SH}^{+} A^{3} \Pi$ state.

\begin{tabular}{lllll}
\hline & \multicolumn{2}{c}{ Normal } & \multicolumn{2}{c}{ Deuterated } \\
Quantity & \multicolumn{1}{c}{ RAS } & Exp & RAS & Exp \\
\hline$R_{e}$ & $1.502 \AA$ & 1.501 & 1.502 & - \\
$D_{e}$ & $1.64 \mathrm{eV}$ & 1.78 & 1.64 & - \\
$D_{0}$ & $1.53 \mathrm{eV}$ & 1.68 & 1.56 & 1.71 \\
$\omega_{e}$ & $1660 \mathrm{~cm}^{-1}$ & 1672 & 1194 & 1201 \\
$\omega_{e} x_{e}$ & $49.1 \mathrm{~cm}^{-1}$ & 47.6 & 26.6 & 24.5 \\
$B_{e}$ & $7.65 \mathrm{~cm}^{-1}$ & 7.66 & 3.95 & 3.95 \\
$\alpha_{e}$ & $0.40 \mathrm{~cm}^{-1}$ & 0.36 & 0.15 & 0.13 \\
$T_{e}$ & $30595 \mathrm{~cm}^{-1}$ & 30345 & - & 30356 \\
\hline
\end{tabular}

\subsection{RASSI}

The routine use of the RASSI program is for calculation of transition moments and similar quantities, such as the life times reported above. However, the program is very useful in a number of less standard contexts. In a recent study of $\mathrm{O}_{4}^{+}$, a strong symmetry-breaking tendency (giving $\mathrm{O}_{2}+\mathrm{O}_{2}^{+}$even in symmetric conformations) was handled by simply using the two symmetry-broken solutions as quasi-diabatic states and computing the hamiltonian coupling elements by RASSI. Rather than reproducing the relevant figures here, we refer the interested reader to the original article (Lindh \& Barnes 1993).

\subsection{CASPT2}

Apart from the two benzene examples presented below, there is a rapidly accumulating body of experience showing that CASPT2 may be one of the most powerful methods to emerge for many years. The interested reader should see also e.g. Roos \& Andersson 1993 or Roos et al. 1993.

Benzene pilot study. The valence electronic spectrum of benzene has been used for a very long lime as a test of ab initio methods. Only recently has reliable results been obtained with an overall accuracy of better than $1 \mathrm{eV}$. The key problem is the large variation in ionicity among the valence states, with a resulting variation in the dynamic correlation, primarily in the form of intramolecular dispersion interaction between the $\pi$ electrons and the $\sigma$ skeleton. The following pilot study demonstrates the problem (Andersson \& Roos 1993). A common misrepresentation is to regard these excited states as primarily single excitations, with an open-shell Hartree-Fock structure. However, in reality, the structure varies strongly, with the ${ }^{1} E_{2 g}$ state as a rather extreme case. In Tab. 4 , note that the multi-excited character of the wave functions would be much more pronounced if e.g. ground state Hartree-Fock orbitals were used. The figures here apply to state-specific optimized CASSCF orbitals. In the second part of 
Table 4. Pilot study: Singlet excited valence states of benzene.

\begin{tabular}{|c|c|c|c|c|c|}
\hline Weight of $e$ & citatio & ns fro & m lea & ling $\mathrm{cc}$ & \\
\hline Exc. type & ${ }^{1} A_{1 g}$ & ${ }^{1} B_{2 u}$ & ${ }^{1} B_{1 u}$ & ${ }^{1} E_{1 u}$ & $E_{1 u}{ }^{1} E_{2 g}$ \\
\hline Singly & & $80 \%$ & $92 \%$ & $87 \%$ & $-48 \%$ \\
\hline Doubly & & $15 \%$ & $3 \%$ & $5 \%$ & $-42 \%$ \\
\hline Triply & & $2 \%$ & - & $2 \%$ & $-3 \%$ \\
\hline Excitation & nergies & $\mathrm{eV}$ & & & \\
\hline Method & ${ }^{1} A_{1 g}$ & ${ }^{1} B_{2 u}$ & ${ }^{1} B_{1 u}$ & 210 & $E_{1 u}{ }^{1} E_{2 g}$ \\
\hline $\mathrm{CAS}(6)$ & & 5.0 & 7.9 & 9.3 & -8.1 \\
\hline +PT2 & & 4.6 & 5.9 & 6.5 & 7.7 \\
\hline $\mathrm{CAS}(12)$ & & 4.9 & 7.4 & 8.8 & 7.7 \\
\hline +PT2 & & 4.7 & 6.1 & 7.1 & 7.7 \\
\hline Exp & & 4.9 & 6.2 & 6.9 & $\begin{array}{ll}7.6 & 7.8\end{array}$ \\
\hline Dynamic c & rrelatic & on con & itribu & ions, e & \\
\hline Corr. type & ${ }^{1} A_{1 g}$ & ${ }^{1} B_{2 u}$ & ${ }^{1} B_{1 u}$ & ${ }^{1} E_{1 u}$ & $E_{1 u}{ }^{1} E_{2 g}$ \\
\hline$\sigma \sigma$ & 12.6 & 12.7 & 12.8 & 12.8 & -12.7 \\
\hline$\sigma \pi$ & 7.7 & 8.0 & 8.8 & 9.6 & -8.0 \\
\hline$\pi \pi$ & 0.9 & 1.0 & 1.8 & 1.8 & $-\quad 1.0$ \\
\hline
\end{tabular}

the table, it is seen how CASPT2 is able to correct for most of the occasionally large errors in the CASSCF energies. Only the six $\pi$-electrons are active. Two active spaces are used: The first comprises the valence $\pi$ orbitals, while the second is twice as large. Note the small improvement to the ${ }^{1} B_{1 u}$ and ${ }^{1} E_{1 u}$ states with the larger active space, and how CASPT2 acts to correct them. Note also how, with the larger space, we begin to see Rydberg intruders (The $2^{1} E_{1 u}$ state). In the last part of the table, the dynamic correlation energies have been broken up into different contributions. The second line can be interpreted as the contributions from the instantaneous response of the sigma electrons to the charge of the $\pi$ electrons; naturally, the effect is larger (by up to $2 \mathrm{eV}$ !) for the states with predominantly ionic structures.

Benzene application example. We finally offer a recent application example: Table 5 presents some of the results of a recently conducted study of singlet and triplet excited states of benzene up to about $7.9 \mathrm{eV}\left(64000 \mathrm{~cm}^{-1}\right)$ (Lorentzon et al. 1993). The basis set was basically our standard basis set of ANO type (Widmark et al. 1990), but augmented with a specially prepared one-center basis to represent the Rydberg orbitals correctly. As can be seen in the tables, the accuracy in vertical excitation energies is generally about $0.1 \mathrm{eV}$.

Acknowledgements. I wish to thank the organizers of this conference for the kind invitation. To me, it seems that this kind of inter-disciplinary symposia is 
Table 5. CASPT2 singlet excitation energies for Benzene.

\begin{tabular}{|c|c|c|c|c|}
\hline State & $\begin{array}{r}\mathrm{E}_{C A S} \\
(\mathrm{eV})\end{array}$ & $\begin{array}{r}\left\langle z^{2}\right\rangle \\
\text { (a.u.) }\end{array}$ & $\begin{array}{r}\mathrm{E}_{P T 2} \\
(\mathrm{eV})\end{array}$ & $\begin{array}{l}\text { Exp. } \\
(\mathrm{eV})\end{array}$ \\
\hline \multicolumn{5}{|c|}{ Valence ${ }^{1} \pi \pi^{*}$} \\
\hline $1^{1} B_{2 u}$ & 4.83 & 30.1 & 4.82 & 4.90 vert \\
\hline $1^{1} B_{1 u}$ & 7.33 & 31.9 & 6.28 & 6.20 vert \\
\hline $1^{1} E_{1 u}$ & 8.45 & 35.1 & 7.09 & 6.94 vert \\
\hline $2^{1} E_{2 g}$ & 7.96 & 29.8 & 7.86 & 7.86 vert \\
\hline \multicolumn{5}{|c|}{ Rydberg ${ }^{1} \pi \pi^{*}$} \\
\hline $2^{1} E_{1 u}$ & 6.48 & 79.1 & 7.17 & 7.41 \\
\hline $2^{1} A_{1 g}$ & 7.19 & 88.3 & 7.76 & $7.81 ?$ \\
\hline $1^{1} E_{2 g}$ & 7.11 & 88.3 & 7.77 & 7.81 \\
\hline $1^{1} A_{2 g}$ & 7.10 & 88.2 & 7.82 & 7.81 \\
\hline \multicolumn{5}{|c|}{ Rydberg ${ }^{1} \pi \sigma^{*}$} \\
\hline $1^{1} E_{1 g}$ & 6.56 & 46.2 & 6.34 & 6.334 \\
\hline $1^{1} A_{2 u}$ & 6.68 & 44.7 & 6.85 & 6.932 \\
\hline $1^{1} E_{2 u}$ & 6.81 & 45.1 & 6.95 & 6.953 \\
\hline $1^{1} A_{1 u}$ & 6.77 & 45.4 & 6.99 & - \\
\hline $1^{1} B_{2 g}$ & 7.30 & 64.9 & 7.54 & $7.460 ?$ \\
\hline $1^{1} \dot{B}_{1 g}$ & 7.30 & 65.2 & 7.54 & $7.460 ?$ \\
\hline $2^{1} E_{1 g}$ & 7.34 & 63.5 & 7.54 & 7.535 \\
\hline $3^{1} E_{1 g}$ & 7.39 & 52.4 & 7.54 & - \\
\hline
\end{tabular}

a fruitful idea. The RASSCF, RASSI and CASPT2 methods reported in this contribution results from work within a joint study contract with IBM Sweden, and supported by the National Research Council of Sweden. They are part of the MOLCAS program package described by Andersson et al. 1991.

\section{References}

Ahlrichs R., Scharf P., Ehrhardt C., 1985, J. Chem. Phys., 82, 890

Almlöf J., Taylor P. R., 1987, J. Chem. Phys., 86, 4070

Andersson K., Fülscher M. P., Lindh R., Malmqvist P.- $\AA$., Olsen J., Roos B. O., Sadlej A. S., Widmark P. O., 1991, MOLCAS version 2. University of Lund, Sweden

Andersson K., Malmqvist P.- $\AA$., Roos B. O., Sadlej A. S., Wolinski K., 1990, J. Phys. Chem., 94, 5483

Andersson K., Malmquist P.- $\AA$., Roos B. O., 1992, J. Chem. Phys., 96, 1218

Chong D. P., Langhoff S. R., 1986, J. Chem. Phys., 84, 5606

Clementi E., Corongiu G., Stradella O. G., 1991, Density Functionals for Molecules, p321 ff in: Modern techniques in computational chemistry: MOTECC-91 (ed. E. Clementi), Leiden 1991.

Gdanitz, R. J., Ahlrichs, R., 1988, Chem. Phys. Letters, 143, 413

González-Luque R., Merchán M., Roos B. O., 1992, Molec. Phys., 76, 201 
Lindh R., Barnes L. A., 1993, J. Chem. Phys., (in press).

Lorentzon J., Malmqvist P.- $\AA$, Roos B. O., 1993, J. Chem. Phys., (submitted).

Malmqvist P.- $\AA$., Rendell A. P., Roos B. O., 1990, J. Phys. Chem., 94, 5477

Malmqvist P.- $\AA$., Roos B. O., 1989, Chem. Phys. Letters, 155, 189

Olsen J., Roos B. O., Jørgensen P., Jensen H. J. Aa., 1988, J. Chem. Phys., 89, 2185

Roos B. O., 1980, Int. J. Quantum Chem. Symp., 14, 175

Roos B. O., Andersson K., 1993, Int. J. Quantum Chem., 45, 591

Roos B. O., Serrano-Andrés L., Merchán M., 1993, Pure Appl. Chem., 65, 1693

Roos B. O., Taylor P. R., Siegbahn P. E. M., 1980, Chem. Phys., 48, 157

Rostas J., Horani M., Brion J., Daumont D., Malicet J., 1984, Molec. Phys., 52, 1431

Watts J. D., Stanton J. F., Bartlett R. J., 1991, Chem. Phys. Letters, 178, 471

Widmark P. O., Malmqvist P.- $\AA$., Roos B. O., 1990, Theor. Chim. Acta, 77, 271 